\title{
Identifying logical evidence
}

\author{
Ben Martin ${ }^{1}$ \\ Received: 10 October 2019 / Accepted: 2 March 2020 / Published online: 13 March 2020 \\ (c) The Author(s) 2020
}

\begin{abstract}
Given the plethora of competing logical theories of validity available, it's understandable that there has been a marked increase in interest in logical epistemology within the literature. If we are to choose between these logical theories, we require a good understanding of the suitable criteria we ought to judge according to. However, so far there's been a lack of appreciation of how logical practice could support an epistemology of logic. This paper aims to correct that error, by arguing for a practice-based approach to logical epistemology. By looking at the types of evidence logicians actually appeal to in attempting to support their theories, we can provide a more detailed and realistic picture of logical epistemology. To demonstrate the fruitfulness of a practice-based approach, we look to a particular case of logical argumentation-the dialetheist's arguments based upon the self-referential paradoxes-and show that the evidence appealed to support a particular theory of logical epistemology, logical abductivism.
\end{abstract}

Keywords Logical abductivism · Logical epistemology · Logical evidence · Practice-based approach · Dialetheism · Self-referential paradoxes

\section{Motivating logical abductivism}

The last 50 years has been marked by a proliferation in the number of formal logical systems to serve a wide spectrum of technical and philosophical purposes. Dynamic logics to model reasoning within mobile robots, temporal logics to model formal verification within systems, and annotated logics to model systems containing uncertainty. Sometimes this proliferation is epistemically unproblematic, as the logics are either simply proposed as being interesting in themselves, or seen to serve a distinct purpose from those of the other available logics. At other times, however, several non-equivalent logics are considered as candidates to serve the same purpose. In such cases, we find ourselves engaged in theory choice.

Ben Martin

Benjamin.martin@uib.no

1 Department of Philosophy, University of Bergen, Postboks 7805, 5020 Bergen, Norway 
Probably the most prominent and philosophically interesting example of such theory choice is over logics that aim to not just solve a certain technical or philosophical problem, but aim to provide a general account of validity - what propositions follow from what. Multiple logics have been proposed as viable candidates to capture validity: paraconsistent logics, intuitionistic logics, paracomplete logics, quantum logics, and of course classical logic. Given that these various logics license different rules of implication, often with significant repercussions, it clearly matters which logic we ultimately endorse. Yet, if we are to make a principled decision over which logic to endorse, we also need an understanding of what constitutes logical evidence, which ultimately requires a theory of logical epistemology. The problem that we currently have when faced with such theory choice is that a fully-formed account of how we come to know logical propositions, and what exactly constitutes logical evidence, is not forthcoming.

While traditional accounts of logical epistemology when it comes to validity have appealed directly to intuitions and definitions, none of these accounts have provided anywhere near a detailed enough explanation of how theory-choice between different logics of validity could occur. Recently, in an attempt to rectify the failures of these accounts, a logical epistemology which has Quine's (1951) empirical holism as its progenitor has been proposed, emphasising similarities between logical and scientific methodology (Priest 2014; Williamson 2017). According to this account of logical epistemology, logical propositions are not directly justified by intuitions or definitions, but rather logical theories are justified by their ability to best accommodate relevant data. In other words, logical theories are justified by abductive means.

Unfortunately, at present, the logical abductivist picture is also incomplete, for three significant reasons. Firstly, apart from appealing to the weaknesses of traditional accounts of logical epistemology, logical abductivism itself presently fails to possess positive evidence. There are no independent reasons to think that logical theories are justified by abductive means. Secondly, even if there is evidence that logical theories are justified by abductive means, there is no explicit agreement over which types of data logical theories are attempting to accommodate. Providing an account of what these data are exactly is obviously important as it will ultimately impact the plausibility and distinctiveness of the logical abductivist proposal. If logical theories are only attempting to accommodate our intuitions about validity, say, then the difference between logical abductivism and traditional rationalist accounts of logical knowledge will be less marked. Lastly, by sharing similarities with Quine's empirical holism, logical abductivism inherits some of the methodological problems of holism. Particularly, what is known as the background logic (or, centrality) problem: in order to justify our logical theory $L$ on the basis of non-direct evidence $E$, we will be required to make logical inferences regarding the consistency of $E$ with $L$, which will require us to presuppose the validity of certain rules of implication $R$ (Shapiro 2000; Wright 1986). What, though, are the grounds on which we are justified in believing that the members of $R$ are valid? Any appeal to abductive criteria will seemingly lead to a 
regress or, eventually, circularity. Consequently, at least some logical beliefs must be justified by non-abductive means. ${ }^{1}$

This paper contributes to answering the first and second concerns above, leaving the third for elsewhere. We propose that by looking to logical practice we have both a means to find positive support for logical abductivism over competing theories of logical epistemology, and pinpoint the types of data that theories of logical consequence must accommodate. In order to demonstrate the fruitfulness of this practice-based approach to logical epistemology, as a case study we consider prominent arguments from the long-standing and vibrant dialetheism debate.

The paper runs as follows. Section 2 outlines the historically prevalent accounts of logical epistemology, logical rationalism and logical semanticism, and compares them to logical abductivism. Section 3 motivates using logical practice to adjudicate between these accounts of logical epistemology. Section 4 outlines the dialetheism debate and the logics motivated by dialetheism. Section 5 considers two famous arguments for dialetheism, based upon the liar and Russell paradoxes, and Sec. 6 proposes that these arguments lend support to logical abductivism. Lastly, Sect. 7 highlights how the practice-based approach should be extended to other logical debates, and importantly to replies to dialetheism.

\section{The state of play}

The historical consensus has been that logical propositions (and inferences) possess two important epistemological properties ${ }^{2}$ :

- Apriority: Logical propositions are justified solely through a priori sources.

- Foundationalism: At least some logical propositions are known by non-inferential means.

While logic is often considered to share its apriority with (at least some) areas of mathematics (Russell 1920: Ch. 18, 1957: pp. 106-107), its foundationalism is a distinguishing property. For while in mathematics we may presume the validity of certain logical inferences in order to construct proofs, this possibility is not open to us when establishing at least some truths in logic without begging the question. This is simply the background logic problem raising its ugly head. Further, significant

\footnotetext{
1 Note, the background logic problem is not only a problem for those who think that empirical evidence could inform logical theory choice. It is a problem for anyone who thinks that certain evidence for logical propositions (and theories) is non-immediate, and thus inferences from the evidence to the theory (and vice versa) are required.

2 A complication that arises when discussing logical epistemology is the distinction between one's justification for holding certain logical beliefs, such as the belief that the disjunctive syllogism is valid, and the justification that one possesses for inferring according to certain rules of implication. The distinction is necessary if we are to both answer the Lewis Carroll problem (Carroll 1895), and explain why those unversed in logical theory are still able to justifiably infer according to modus ponens. As a consequence, there are two distinct but connected ongoing projects within the epistemology of logic: (1) establishing what justifies our logical beliefs, and (2) establishing what warrants our inferring according to certain fundamental rules of implication (see Boghossian 2000). Our discussion here is firmly embedded within the former project, while we acknowledge that the results could impact the latter. With this in mind, in what follows we will speak in terms of being justified in believing logical propositions (or, theories).
} 
projects within the philosophy of logic, such as Frege's logicism, have presumed that logical knowledge is privileged in its foundational status. While we are told that many arithmetical truths lack self-evidence and thus require proof (Frege 1952: p. 164), in comparison the primitive logical laws are self-evident (Frege 1965: p. xvii) and so require no further argument. Fundamentally, the message from the background logic problem and Frege's logicism is the same: evidence for (at least some) logical propositions is direct in a way in which evidence for other propositions is not.

Combined, the purported apriority and foundationalism of logical evidence has led to two theories of logical knowledge dominating the history of logical epistemology: logical rationalism and logical semanticism (Martin and Hjortland 2020). Both agree that sensory data cannot justify our logical beliefs and that knowledge from other research areas cannot provide evidence for logical propositions, while disagreeing on the source of this non-inferential a priori evidence. While logical rationalists propose that logical knowledge is the result of direct mental insight, such that having the intuition that $p$ is sufficient (if defeasible) evidence for $p$ (Bealer 1998; BonJour 1998), logical semanticists propose that appropriately understanding the meaning of a logical proposition $p$ is sufficient evidence for its truth or falsity (Ayer 1936; Carnap 1937). The question for both parties, therefore, is not whether logical evidence is a priori or non-inferential, but whether it is the result of a quasi-perceptual cognitive faculty or linguistic proficiency.

According to the logical rationalist, logical evidence can be exhaustively accounted for in terms of an immediate awareness of the truth of a logical proposition, or the validity of an inference, using a quasi-perceptual sense. These mental insights (or, intuitions), in virtue of representing states of affairs, are able to provide us with evidence for the truth or falsity of propositions about their contents (Chudnoff 2011), including logical propositions:

When you have an intuition that $A$, it seems to you that $A$... [understood as a] genuine kind of conscious episode. For example, when you first consider one of de Morgan's laws, often it neither seems true nor seems false; after a moment's reflection, however, something happens: it now just seems true. (Bealer 1998: p. 207)

So, we simply non-perceptually see that the relevant logical proposition is true.

The logical semanticist, in contrast, denies the need to posit a new cognitive faculty in order to accommodate logical knowledge. Instead, we come to know the truth or falsity of logical propositions simply by understanding the meaning of their constituent parts:

If one knows what is the function of the words 'either', 'or', and 'not', then one can see that any proposition of the form 'Either $\mathrm{p}$ is true or $\mathrm{p}$ is not true' is valid. (Ayer 1936: p. 79)

In other words, logical propositions are epistemologically analytic (Boghossian 1996). ${ }^{3}$ For certain logical semanticists, such as the logical positivists, the epistemological analyticity of logical propositions is a direct consequence of the view that

\footnotetext{
3 Note, logical semanticism does not itself privilege any particular theory of meaning. Thus, to take two particularly common theories of meaning in the literature, the meaning (and thus analyticity) of these logical
} 
logical propositions are true (or false) solely in virtue of the meaning of their constituent parts (Ayer 1936: p. 73). In other words, they are metaphysically analytic (Boghossian 1996). This latter view itself is a consequence of attempting to accommodate the necessary truth of logical propositions without postulating any dubious notion of metaphysical necessity (Carnap 1963: p. 46). If logical propositions are necessarily true, this is solely a product of linguistic conventions on meaning, rather than the world.

Logical semanticists need not be committed to metaphysical analyticity, however. While metaphysical analyticity entails epistemological analyticity, the inverse is not true. It is coherent to suggest that one can recognise the truth of 'All Belgians are Europeans' by simply understanding the meaning of its constituent parts without admitting that its truth is due solely to its meaning, rather than facts about Belgium and Europe (see Boghossian 1996; Williamson 2007: Ch. 3). Rather, modern advocates of logical semanticism are motivated into the position by a commitment to logic's foundationalism and a deep suspicion of intuition (see, for example, Boghossian 2000, 2001).

Both of these traditional accounts of logical epistemology face considerable criticisms, often due to the sources of evidence they propose. Logical rationalism has been accused of answering the mystery of a priori knowledge with yet a further mystery, that of intuition (Boghossian 2000: p. 231). In comparison, logical semanticism faces classic Quinean (1951) concerns over the significance of any distinction between analytic and synthetic propositions, concerns over the indeterminacy of meaning (Giaquinto 2008), and evidence that linguistic competence is never sufficient to entail the acceptance of a proposition, as further theoretical commitments can block assent (Williamson 2007: Ch. 4).

It is against the backdrop of these perceived problems facing logical rationalism and semanticism that logical abductivism has grown in support. According to the theory, we come to be justified in believing a logical proposition $p$ not by either directly intuiting its truth, or understanding its constituent parts, but rather by being justified in believing a logical theory $L$ containing $p$. Further, we come to be justified in believing a particular logical theory $L$ because it better accommodates the relevant data, and possesses more relevant theoretical virtues, than other available theories:

Given any theory, in science, metaphysics, ethics, logic, or anything else, we choose the theory which best meets those criteria which determine a good theory. Principal amongst these is adequacy to the data for which the theory is meant to account. In the present case, these are those particular inferences that strike us as correct or incorrect...Adequacy to the data is only one criterion, however. Others that are frequently invoked are: simplicity, non-(ad hocness), unifying power, fruitfulness. (Priest 2014: p. 217)

The influence of Quine's (1951) empirical holism on logical abductivism is clear. We cannot hope to have some direct privileged access to the truths of validity any

Footnote 3 continued

propositions could equally be explained in terms of their truth-conditions or their inferential relationship to other propositions.

4 Other prominent supporters of logical abductivism include Peregrin and Svoboda (2017), Russell (2014, 2015) and Williamson (2017). 
more than we can to the truths of the natural sciences. The foundationalism of logical rationalism and semanticism is fundamentally flawed.

We should not presume, however, that the position of the contemporary logical abductivist is identical to Quine's. There are two notable points of difference. Firstly, due to his naturalism, Quine is committed to observational data serving as the final arbiter for all theories, including logical theories (Quine 1986: p. 100). Yet, nothing within the contemporary logical abductivist's theory requires her to be a naturalist. Indeed, as can be seen from Priest's quote above, some modern logical abductivists prioritise a priori evidence in the case of logic. ${ }^{5}$ Not all modern logical abductivists take logical evidence to be primarily a priori, however. Williamson (2017: p. 13), for example, is quite clear that "independently well-confirmed sentences, such as wellestablished principles of physics" constitute part of the data which logical theories must accommodate. Here, then, there is potential disagreement among contemporary logical abductivists.

Secondly, Quine (1986: p. 100) stressed the importance of conservativeness when making alterations to our overall theories in the face of recalcitrant data, with his principle of minimal mutilation. This requires only changing our logical theory, rather than other parts of our web of belief, in drastic circumstances. It was exactly this conservativism which allowed Quine to justify his continued support for classical logic despite the significant challenges posed by vagueness and the logico-semantic paradoxes. Again, there is no need for the abductivist to follow Quine here. In fact, there is good evidence to think they do not. One can hardly propose that Priest's (2006b) advocation of dialetheism, and rejection of classical logic, is a conservative revision in the face of the troublesome logico-semantic paradoxes. There are more conservative solutions available, as we shall see below. Additionally, Williamson (2017), although he shares Quine's commitment to classical logic, denies that conservatism plays any role in his continued support. This does not mean, of course, that contemporary logical abductivists agree on the theoretical virtues for logical theories. While all of Williamson (2017: p. 14), Priest (2014: p. 217) and Russell (2015: p. 800) mention strength, simplicity and unifying power as theoretical virtues, they diverge in others they mention (Williamson is unique in mentioning elegance, for example), and of course there is no assurance from mere lists that they share the same conceptions of these virtues.

These differences between Quine and contemporary logical abductivists (and between contemporary logical abductivists themselves), highlight how abductivists can agree on the basic mechanism of logical justification while disagreeing on which data logics must accommodate, and which theoretical virtues can be appealed to when the relevant data inevitability underdetermines theory choice. ${ }^{6}$ Thus, even if we have

\footnotetext{
5 See also Peregrin and Svoboda (2017: Ch. 6 and 8), who consider judgements about ordinary vernacular arguments to be the primary source of evidence for logical theories.

${ }^{6}$ For the sake of brevity, and maintaining the focus of the current paper, we have spent less time than we could have here considering in detail the differences between the available versions of logical abductivism in the literature. For example, Peregrin and Svoboda (2017: p. 11) speak of logical theories resulting from a process of reflective equilibrium, rather than abduction. While we think that ultimately their account of logical methodology is abductive, given their recognition that it is whole logical theories which are evaluated (Peregrin and Svoboda 2017: Ch. 10), it's an interesting question in general what substantial differences there could be between proposing that logical epistemology operates by reflective equilibrium
} 
good reason to think that logical theories are justified by abductive means, we still require details on which data exactly logics must accommodate.

Further, discussion of logical abductivism has so far concentrated on how logical theories could be selected for on the basis of abductive criteria, the weaknesses of foundationalist accounts of logical epistemology, and how we should conceive of the theoretical virtues of logic. Limited positive evidence has been provided in support of logical abductivism. Priest (2014), for example, shows how logical theories could be chosen by abductive means, using a Weighted Aggregate Model (WAM). However, this neither shows that logical theories are chosen on the basis of abductive considerations, nor that they ought to be.

By looking to logical practice we aim to be able to go some way to answering these two significant questions of logical abductivism: to not only find some positive support for logical abductivism, but discover the types of data logics must accommodate by looking to the types of arguments logicians actually appeal to within debates.

Before we move onto considering how logical practice could support logical abductivism, one further important clarification is required. Some advocates of the view that logical theories are supported by abductive means also explicitly endorse the view, known as anti-exceptionalism about logic, that logical knowledge and methodology is not exceptional, and shares certain features with the sciences. The clearest example of this view is found in Hjortland (2017: p. 632):

Logic isn't special. Its theories are continuous with science; its method continuous with scientific method. Logic isn't a priori, nor are its truths analytic truths. Logical theories are revisable, and if they are revised, they are revised on the same grounds as scientific theories. These are the tenets of anti-exceptionalism about logical theories. ${ }^{7}$

It is important to appreciate that anti-exceptionalism about logic so understood is not synonymous with logical abductivism. Fundamentally, logical anti-exceptionalists propose a similarity between the methodology of logic and the sciences which the logical abductivist need not. For example, the logical abductivist could be completely non-committal about the methodology of the sciences, and its similarity to logic, so that their thesis is solely about the epistemology (and, methodology) of logic. Further the logical abductivist could deny that certain important features of scientific methodology, such as the role of predictions in testing theories, are found within logic. Such a difference between logical and scientific methodologies would not call into

\section{Footnote 6 continued}

and by an abductive method. However, we leave consideration of these potential differences to elsewhere. The important question for the current paper is which methodology and sources of data does logical practice imply are suitable for the epistemology of logic. We thank an anonymous referee for pushing us on this point.

7 Note that while this is the most often cited articulation of anti-exceptionalism about logic in the literature, one should not take the theses contained as definitional of the position. For example, if the fundamental claim of the position is that the methodology of the sciences and logic are similar in important respects, then we may have good reason to disagree with Hjortland that logical evidence cannot be a priori, for we may insist that the sciences also rely upon some a priori evidence. How we should appropriately understand anti-exceptionalism about logic is an important question for contemporary epistemology of logic, but is ultimately irrelevant for the present paper's goals, and well beyond its scope. We use the present quote merely as a popular articulation of the position. Thanks to an anonymous referee for raising this point. 
question the viability of logical abductivism, only a certain interpretation of antiexceptionalism about logic. Inversely, the logical anti-exceptionalist need not be an abductivist, for they may be convinced that prediction plays a special role within scientific methodology such that "fitting" existent data isn't enough to support theories (Mayo 1991). Consequently, in virtue of being an anti-exceptionalist about logic, they also commit themselves to a form of logical predictivism, rather than abductivism. Importantly for this paper, given that logical abductivism and anti-exceptionalism about logic are not synonymous, in finding evidence for logical abductivism, we are not automatically providing evidence for the truth of anti-exceptionalism about logic. In order to be justified in believing anti-exceptionalism about logic, in addition, we would need reason to believe that scientific methodology operated by the same mechanisms. This latter claim will not be of interest to us here, as we obviously would not expect to find evidence for it merely by looking at logical practice. With this in mind, let us move onto how debates over logical epistemology can hope to learn from logical practice.

\section{Learning from practice}

An assumption of this paper is that we can learn about logical epistemology by looking to the actual practice of logicians. What justifies this assumption? The simple answer is that the same considerations hold in the case of logic as they do in the empirical and mathematical sciences. It is well recognised that the philosophy of science, and its conclusions about scientific methodology and epistemology, were in an unhealthy state until the actual practice of scientists was taken seriously (see, for example, Hacking 1983; Burian 2001). One cannot be expected to make sound conclusions about how we come to be justified in believing scientific theories without taking notice of how scientists go about justifying their own theories (and indeed go about experimenting generally). The same can be said for mathematics, and the last 20 years has seen a far greater appreciation of mathematical epistemology by looking in detail at the practice of working mathematicians [see, for example, Giaquinto (2007), Van Bendegem (2003), and Van Kerkhove and Van Bendegem (2008)]. The assumption underlying all of these uses of a practice-based approach to the epistemology of a particular field is that scientists and mathematicians are generally very good at recognising what constitutes suitable evidence for a theory within their field, and what the plausible means are to justify a proposition or theory.

Of course, these considerations hold not only for the empirical sciences and mathematics. Any theory of jurisprudence which did not take into consideration the practice of legal professionals would seem empty of necessary details. The assumption in all of these cases is the same. Experts within a field $F$ are reliable guides to what constitutes evidence within $F$, and we have no reason to think the same isn't true of logicians. Logicians supply suitable, if defeasible, reasons for their theories. Consequently, the reasons that logicians supply for their theories should constitute a reliable guide to the epistemology of logic, just as looking to the experimental practice and arguments of empirical scientists provides a reliable guide to the epistemology of the empirical sciences. 
If all of this is correct, and we will not spend any longer here arguing for the assumption, then we can use the arguments found within logical disputes as data to evaluate the plausibility of various theories of logical epistemology. Any theory that fails to take seriously a significant portion of practice must be considered deficient. Inversely, any theory that accommodates important elements of practice has an advantage over competitors. Thus, we can use logical practice in order to build an abductive justification for a theory of logical epistemology, and the less able the competing theories are to capture such practice, the stronger this abductive case will become. For example, if we find that logical disagreements very rarely hinge upon disagreements over definitions of logical terms, or intuitions about the target propositions, then this must count heavily against those theories of logical epistemology that require definitions or intuitions to play a pivotal role within the epistemology of logic.

With this in mind, before we move onto considering an instance of logical practice, it will be useful to suggest what we should expect to find within logical debates if the respective theories of logical epistemology were correct. Take first logical rationalism. According to logical rationalism, we gain justification for our logical beliefs directly from intuitions regarding a particular proposition. We simply see that the proposition $p$ is true or false. Consequently, given logical rationalism, we would expect logical arguments to be full of appeals to intuitions, especially when it comes to certain fundamental propositions on which the remainder of one's logical theory rests. Similarly, when there is disagreement between logicians over the truth of important claims, we ought to find each side appealing to their intuitions over the truth of the relevant claims. Indeed, for the logical rationalist there is nothing else the logician can appeal to. If another party disagrees with us, all we can do is suggest that our interlocutor is not having the right kinds of intuitions, and that they should look a bit harder. It is just such attitudes that parties within a logical debate ought to have towards one another if logical rationalism is true.

The logical semanticist is in a similar position. According to her, we gain justification for our logical beliefs directly by grasping the meaning of logical propositions. Once we understand the constituent parts of the proposition, we are immediately justified in believing it to be true. Thus, if there were any disagreement between logicians over the truth of certain logical propositions, we would expect to find each side appealing to the meaning of the relevant propositions in order to substantiate their own claims to its truth or falsity. Again, there is nothing other than the meaning of the propositions for the logician to appeal to. If one's interlocutor fails to assent to the same propositions as us, we are committed to saying this is simply because she has misunderstood its content, and is talking about a different matter than we are. All we can do in this case, in hope of rescuing the situation, is to point out the proposition's meaning even more explicitly. It is just such interactions we would expect within a logical disagreement if logical semanticism were true. ${ }^{8}$

\footnotetext{
8 Could, in addition, according to logical semanticism, the logician answer their interlocutor by appealing to the way the logical community generally use a logical term? This depends. If this appeal takes the form of simply pointing out the definitions of the relevant term that other members of the community use, then yes this is what semanticism would expect and sanction. This would be a case of simply showing that the majority of the community agree with your understanding of the term, rather than your interlocutor's. If, however, instead the logician's appeal took the form of referring to the type of inferences which logicians
} 
Lastly, what about logical abductivism? According to logical abductivism, we judge the adequacy of a theory by its ability to accommodate the relevant data, and subsequently come to be justified in believing a logical theory by evaluating the degrees to which each available theory fits the data. Thus, if logical abductivism were correct, we would expect logical arguments to be full of appeals to well-recognised data which it is commonly accepted within the community that theories ought to be able to accommodate. We ought to also expect explanations of how the practitioner's theory can accommodate the data, and potentially solve recognised puzzles within the field. Nothing so far has been said about what these data are, or what exactly it is for theories to fit such data in the case of logic. It is just such details we might hope logical practice can help us answer if logical abductivism has any plausibility at all over its competitors.

With a clearer sense of how logical practice might provide some insight into the correct theory of logical epistemology, and what we should expect if any of the three epistemologies of logic considered are true, we can move to looking at a particular case of logical disagreement, and test the fruitfulness of the approach. Here we look to the dispute between dialetheic and non-dialetheic logicians over the truth of contradictions, and particularly the dialetheist's arguments based upon the self-referential paradoxes. The choice is motivated by the debate's longevity and prominence. Both have ensured that there is a considerable amount of data to use in forming a picture of logical practice. It is no good using as a case study a debate which hasn't yet had time to play out. In so choosing the debate, we leave significant scope for further enquiry (as detailed in Sect. 7). The dialetheism debate is not the only choice which fits these criteria, but it is a suitable choice. We begin by providing an overview of what dialetheism proposes, and the logics it recommends.

\section{A case study: the dialetheism debate}

Dialetheism is the thesis that some contradictions are true (Priest 2006a: p. 1). While the theory has been motivated by a whole host of reasons, including inconsistent obligations and metaphysical considerations (Priest 2006b: Ch. 11-13), it is those arguments based upon the self-referential logico-semantic paradoxes that have gained the greatest purchase within the literature. Particularly prominent examples are the liar and Russell paradoxes, and this prominence will be reflected in our examination of the arguments below.

Dialetheism isn't then itself a logic. However, the theory does argue for a change of logic from the classical orthodoxy. Indeed, if dialetheism is to be logically sustainable,

\footnotetext{
Footnote 8 continued

(and, potentially, others) generally accept when using the relevant term, in order to justify their claim about its meaning, then this would be beyond the type of evidence sanctioned by semanticism. For being aware of these inferences generally accepted by the community does not provide us directly with an account of the meaning of the relevant terms and associated propositions. To gain an account of their meaning, one would need to draw inferences from these instances to construct a theory of what the meaning of the terms might be given these accepted inferences. In such a case, logical evidence becomes again inferential (and thus non-foundational), the background logic problem raises its head, and we have in effect a version of logical abductivism which treats as its data the inferences found acceptable by logicians (and, potentially, others), instead of logical semanticism. We thank an anonymous referee for highlighting this point.
} 
these changes are required. What kind of logic, exactly, does dialetheism require, and recommend as a theory of validity?

There are two essential properties any logic $L$ must possess if it's to accommodate dialetheism:

(Di) $L$ must contain some interpretations in which some contradictions are at least true, with contradictions conceived of as the conjunction of a proposition and its negation

(Dii) $L$ must be strongly paraconsistent $\left(\{A \wedge \neg A\} \nvdash_{L} B\right)$

The rationale for a dialetheist requiring her logic to fulfil conditions (Di-ii) are straightforward. Firstly, without condition (Di), the dialetheist's logic would not be able to successfully model true contradictions, with formulae of the form $A \wedge \neg A$ treated as symbolisations of contradictions.

Secondly, once it's acknowledged that the dialetheist's logic must fulfil condition (Di), it's necessary that the logic also fulfils condition (Dii) unless the dialetheist is to be committed to trivialism, the thesis that every proposition is true. While some authors have argued in favour of trivialism (Kabay 2008), this isn't true of dialetheists themselves. Members of the dialetheist research programme think they have good reason to deny the truth of trivialism, while admitting that some contradictions are true (Priest 2006a: Ch. 3). ${ }^{9}$ The combination of these commitments, then, requires that contradictions do not explode, and hence the need for condition (Dii) to be fulfilled by a suitable logic.

For simplicity, call any logic that fulfils conditions (Di-ii) a diatheically suitable logic. While every non-trivial dialetheist must endorse such a logic, this doesn't require every logician who advocates a diatheically suitable logic to be a dialetheist. Indeed, some famous advocates of such logics are not dialetheists. Take Newton da Costa (1974), for example, whose $C_{i}(1 \leq i \leq \omega)$ logics fulfil (Di-ii), but who does not commit himself to true contradictions (da Costa 1982). Such non-dialetheic advocates of these logics may endorse them because they serve a practical purpose, or they may provide a non-standard (in other words, non-dialetheic) interpretation of the logics, such as in terms of belief states. We, however, are interested exclusively in those who argue for a diatheically suitable logic on the grounds of dialetheism.

While (Di-ii) are the essential properties for any dialetheist's logic, they are not the only properties which a dialetheist may desire of her logic. These further properties may flow out of independent philosophical motivations, or be tied firmly to the dialetheist's arguments for dialetheism. Here are some of the further properties found in the literature which dialetheists desire for their logic:

(Diii) Propositional parameters are true and false within some interpretations of $L$ (Div) $L$ respects the normal semantics for the Boolean connectives. ${ }^{10}$

\footnotetext{
9 Of course, all trivialists are committed to dialetheism, for the simple reason that they admit every proposition to be true, including contradictions. However, this doesn't mean that trivialists are members of the dialetheic research programme, which requires that they share with dialetheists similar arguments and aims. Our interest here is with the dialetheist's arguments, and particularly how they argue for a new theory of validity.

10 That is, Conjunction: $v(A \wedge B)=\min \{v(A), v(B)\}$; Disjunction: $v(A \vee B)=\max \{v(A), v(B)\}$; and Negation: $v(\neg A)=1-v(A)$. In some cases, dialetheists now use a relational semantics for their logics (as
} 
(Dv) $L$ is paraconsistent $(\{A, \neg A\} \not \models B)$

(Dvi) $L$ can tolerate the inclusion of a transparent truth predicate without trivialising What's the motivation for requiring one's logic to have these properties? (Diii) is motivated directly by the dialetheic solutions to certain semantic paradoxes. For example, according to the dialetheic treatment of the simple liar sentence:

( $\lambda)\ulcorner\lambda\urcorner$ is false

$(\lambda)$ is both true and false, which subsequently entails a true contradiction given certain assumptions about the behaviour of conjunction and negation (ensured by (Div)). Thus, $(\lambda)$ is primarily a glutty sentence, which then entails a true contradiction. ${ }^{11}$ Given that it is just these paradoxes which motivate dialetheism for many dialetheists, incorporating (Diii) into their logic is a necessity.

In comparison, (Div) is independently motivated. For example, we find Priest and Routley (1989: pp. 158-9) criticising both Jaśkowski's (1969) discursive $\mathrm{D}_{2}$ logic for not containing a conjunction operator, as none of its operators validate adjunction, and da Costa's (1974) $\mathrm{C}_{i}(1 \leq i \leq \omega)$ logics for not containing a negation which ensures that a proposition $p$ 's negation is true if and only if $p$ is false (Priest and Routley 1989: pp. 164-5). As far as Priest and Routley are concerned, validating adjunction is a necessary condition for a conjunction operator, and a negation operator $\neg$ must ensure that all propositions of the form $A \wedge \neg A$ are logically false (though they may also be true in some interpretations, as in Priest's (1979) LP). Consequently, as the putative negation operator for the $\mathrm{C}_{i}(1 \leq i \leq \omega)$ logics doesn't fulfil this property, "da Costa negation is not negation" (Priest and Routley 1989: pp. 164-5). This commitment to (Div) seems philosophically distinct from the other commitments the dialetheist takes on in virtue of her arguments for dialetheism. Yet, once one commits oneself to (Div), the commitment to (Dv) follows immediately given (Dii). If the dialetheist desires a non-trivial logic, and thus $L$ must be strongly paraconsistent, given that (Div) commits the dialetheist to simplification, $L$ must also be paraconsistent to block triviality.

Lastly, we come to (Dvi). As with (Diii), the dialetheist's commitment to (Dvi) is a direct consequence of their arguments for dialetheism. As we will see below, in arguing for a dialetheic solution to the liar paradoxes, the dialetheist suggests that all non-dialetheic solutions which solve the paradoxes by restricting the transparency of the truth predicate do so at the cost of deeming certain natural language sentences meaningless. Given that this is unacceptable for the dialetheist, they admit a transparent truth predicate into their own logic. However, with the Curry (1942) paradox, it's well known that any logic containing a transparent truth-predicate and a contractive conditional will trivialise. This means that the dialetheist will need to introduce a noncontractive conditional into their logic if it's to contain a transparent truth predicate without trivialising. Of course, not all dialetheists agree on what this conditional should

Footnote 10 continued

first presented in Dunn 1976), rather than a truth-functional semantics (see Priest 2006b: Ch. 19). However, in this case too, the clauses for the Boolean connectives in the dialetheist's logic are expected to match those of classical logic, with the dialetheist's logic diverging from classical logic in allowing for truth-value gluts (see Martin 2018).

11 Note, this isn't true for all liar sentences. Some, such as the strengthened liar discussed below, entail a contradiction, but are only shown to be glutty sentences subsequently given certain assumptions regarding truth and falsity. 
look like (see, for example, Priest 2006b; Beall 2009); but, all agree that (Dvi) is a desideratum for their logic.

The importance of outlining both the essential and desirable properties for a dialetheist's logic is not only that it clarifies which logics exactly a dialetheist would find acceptable, and how such logics differ from other prominent logics within the literature, such as classical and intuitionist logics, but it emphasises the scope of choice which the dialetheist has available to her. Not every logic which fulfils one of the essential criteria for a dialetheist's logic fulfils the other. For example, Bryson Brown's (1999) preservation logic is strongly paraconsistent without fulfilling (Di), by preserving levels of ambiguity with its consequence relation rather than truth. However, there are still a multitude of logics which fulfil both essential conditions that the dialetheist can choose from, including Priest's (1979) LP, da Costa's (1974) $\mathrm{C}_{i}(1 \leq i \leq \omega)$ logics, and the propositional fragment of Batens and De Clercq's (2004) CLuNs. Even if the dialetheist does sign up to all of (Diii-Dvi) as desiderata of their logic, there are still choices to be had. While da Costa's $C_{i}(1 \leq i \leq \omega)$ logics won't fit the bill, as they fail to meet condition (Div), both $\mathbf{L P}$ (with a suitable conditional) and CLuNs will, for example.

This leads us onto the important point when considering prominent dialetheic arguments. Dialetheists very rarely argue directly for a particular logic. They are not throwing all their eggs into one basket. They have a group of acceptable logics in mind. Thus, if an opponent shows that one of these logics is deficient for a particular reason, this should not be of concern to the dialetheist. For the criticism to be truly damaging, one would need to show how this weakness affects all of the logics which fit the dialetheist's desiderata. Consequently, the debate between dialetheists and nondialetheic logicians, whether this be classical or other non-classical logicians, is not a simple debate over which of two logics are correct. There are multiple logics that the dialetheist can resort to. It is, therefore, a debate between research programmes, which necessitates a choice of logic, but not necessary a particular logic. It simply has to be a logic which fits the desiderata of the research programme, which is very rarely just one logic.

With a better understanding of dialetheism, and the logic(s) which the position recommends, let us now move onto considering the dialetheist's arguments for her support of logics with (at least) properties (Di-ii). As has been mentioned, a whole plethora of reasons have been proposed for why we ought to be dialetheists, including inconsistent obligations and the concept of motion. Here, however, we will concentrate on just two of Graham Priest's (2006b) famous arguments for dialetheism: the liar paradoxes and the Russell set.

This, of course, ensures that our discussion here will only be a partial analysis of the types of arguments logicians use to motivate their logic. But, it was always bound to be. Not only will future work be required to complete the picture of the reasons advanced within the dialetheism debate by both sides, but it is necessary that other prominent debates are similarly analysed to ensure that the type of reasons given within the dialetheism debate are representative of those given by members of the logic community as a whole, and thus suitable to generalise upon. However, we must start somewhere, and the prominence of these dialetheic arguments make them apt for analysis. 


\section{Priest's arguments for dialetheism}

In this section we outline Priest's two most famous, and influential, arguments for dialetheism: the putative contradictions that result from the liar paradoxes and the Russell paradox. While one of these arguments is due to the paradoxical nature of semantic properties (particularly within our natural languages), the other is about the paradoxical properties of one of our fundamental mathematical concepts. By looking at how Priest attempts to support dialetheism by appealing to these arguments, and criticises other putative solutions, we can build a more informed picture of the types of evidence logicians appeal to in support of their logics.

\subsection{The liar paradoxes}

As has been known since the work of Tarski (1944: pp. 348-9), troublesome liar-like sentences can arise in any language which is semantically closed. That is, any language $L$ that fulfils the following conditions:

(Si) Any sentence $s$ in $L$ can be named by a term $t$ belonging to $L$, and

(Sii) $L$ 's own semantics can be expressed within the language (e.g., that sentence $s$ is true)

Even more worryingly, however, Tarski also showed that when combined with the intuitively plausible unrestricted T-schema,

$$
T(\ulcorner A\urcorner) \equiv A
$$

and certain rather uncontroversial rules of implication, it can be shown that any semantically closed language $L$ is inconsistent!

Take as an example the strengthened liar:

$(\delta)\ulcorner\delta\urcorner$ is not true

Once we admit $(\delta)$ into our language and the truth of the unrestricted T-schema, it's simple to show by classically valid rules that a contradiction follows:

\begin{tabular}{lll}
\hline$\delta \equiv \neg T(\ulcorner\delta\urcorner)$ & & (L1-Strengthened Liar) \\
$T(\ulcorner\delta\urcorner) \equiv \delta$ & & (L2-Instance of T-Schema) \\
$T(\ulcorner\delta\urcorner) \equiv \neg T(\ulcorner\delta\urcorner)$ & & (L3-From L1-L2 by transitivity) \\
$T(\ulcorner\delta\urcorner) \vee \neg T(\ulcorner\delta\urcorner)$ & & (L4-Instance of LEM) \\
$T(\ulcorner\delta\urcorner) \wedge \neg T(\ulcorner\delta\urcorner)$ & & (L5-From L3-L4 by cases and adjunction) \\
\hline
\end{tabular}

Now, not only is it quite troubling for the non-dialetheic logician that it's possible to show that any seemingly acceptable semantically closed language is inconsistent (given the T-schema and the logical principles above), but given that we have good reason to think that natural languages are semantically closed, the liar paradox puts pressure on the view that no contradictions are true. After all, the argument above seems to show that our natural languages contain just such true contradictions! 
To avoid this unsavoury consequence, the non-dialetheic logician must block the conclusion somehow. But, what are the options? There are three: (1) restrict semantic closure for all non-trivial languages; (2) deny the universal applicability of the Tschema; or (3) reject the validity of one of the classically valid rules used to derive L5. It is just such options we find non-dialetheic logicians exploring in the literature. Paracomplete logicians, such as Field (2008), have attempted to block L5 by rejecting one of the classically valid principles, such the Law of Excluded Middle appealed to in L4. In comparison, some classical logicians have attempted to block the result by restricting the applicability of the T-schema to well-founded sentences, so that the T-schema only applies to those sentences $A$ that satisfy $T(\ulcorner A\urcorner) \vee T(\ulcorner\neg A\urcorner$ ) (Kripke 1975; McGee 1991). Finally, some classical logicians have attempted to rescue the logic from this troubling result by restricting semantic closure.

The architect of this final approach was Tarski (1944) himself, who sought to block L5 by precluding the occurrence of liar sentences within the language $L$ in the first place, by restricting $L$ 's semantic closure. This would be achieved by splitting $L$ into a hierarchy of languages such that, for any language $L_{\mathrm{n}}$, no term within $L_{\mathrm{n}}$ could apply a semantic property to sentences within $L_{\mathrm{n}}$. In terms of truth, this ensures that $L_{\mathrm{n}}$ could not express that sentences within its own language were true. Instead, to express the semantic properties of sentences within $L_{\mathrm{n}}$, a metalanguage $L_{\mathrm{n}+1}$ would be needed. Obviously, in order to ensure every language could have semantic properties applied to it, such an introduction of metalanguages would have to continue ad infinitum. At the cost of postulating an infinite hierarchy of languages, however, the approach restricts the semantic closure of any given language $L$, and ensures that no liar sentence can occur within any of the languages. After all, no sentence within any of the languages can say of itself that it is false or untrue.

As far as Priest is concerned, all of these non-dialetheic approaches fail as adequate solutions to the paradox for at least one of the following three reasons:

(i) The putative solution unnecessarily restricts the expressibility of natural languages.

(ii) The putative solution is incomplete, as it is subject to revenge versions of the liar.

(ii) The putative solution is ad hoc (that is, lacks independent motivation).

In comparison, the dialetheic solution, which is simply to accept the initially unsavoury conclusion at L5 that $(\delta)$ is both true and not true, fails to exhibit any of these theoretical deficits. As it turns out, Tarski's putative solution seemingly falls foul of all three concerns, and so we can use it to detail each of Priest's criticisms of non-dialetheic solutions to the liar.

Firstly, Priest proposes that multiple non-dialetheic solutions have the unfortunate consequence of deeming many obviously meaningful natural-language sentences meaningless. In the case of Tarski's solution, this consequence arises due to directly restricting the semantic closure of languages. By proposing that only the metalanguage $L_{\mathrm{n}+1}$ can contain the truth predicate for sentences in $L_{\mathrm{n}}$, not only does this preclude the meaningfulness of troublesome liar sentences, but totally innocuous sentences like:

( $\xi)$ Every sentence in the article "Identifying Logical Evidence" is true

After all, $(\xi)$ applies the truth predicate to itself. Even if one has concerns over the meaningfulness of all self-referential sentences, and Priest (2006b: pp. 14-15) presses 
the point that we have no independent reasons to be dubious of the meaningfulness of self-referential sentences in and of themselves, then it's possible to construct obviously meaningful pairs of sentences that the advocate of the hierarchy of languages approach must insist are meaningless when combined:

(Trump) Everything that Robert Mueller says is false.

(Mueller) Everything that Donald Trump says is false.

According to the hierarchy of languages approach, either (Trump) or (Mueller) must be part of the metalanguage of the other. Yet, this ensures that the statement from the lower level in the hierarchy (whichever that turns out to be) must be meaningless, although both claims are clearly meaningful. In comparison, by not restricting the semantic closure of English, the dialetheic solution respects natural languages' expressibility.

Secondly, in addition to deeming many meaningful sentences containing semantic concepts meaningless in an attempt to avoid the paradoxical consequences of a liar sentence, Priest proposes that the putative solutions do not even escape all versions of the paradox. Even if the non-dialetheist is able to avoid one particular flavour of the liar, by deeming it meaningless or gappy, the causes of the paradox run so conceptually deep that it is always possible to formulate a version of the liar in the chosen language of the solution, which the putative solution cannot help but admit is meaningful and contradictory. In the case of the hierarchy of languages solution, this means appealing to the truth-value that a sentence has at its level:

$(\gamma)\ulcorner\gamma\urcorner$ is not true at its level

According to Tarski's analysis, $(\gamma)$ must be on some particular level of the hierarchy. Call it $l$. Thus, $(\gamma)$ in effect expresses the bi-conditional that,

$$
T_{l}(\ulcorner\gamma\urcorner) \equiv \neg T_{l}(\ulcorner\gamma\urcorner)
$$

Given the T-schema and the same logical principles appealed to above, we get a contradiction.

The only option available to the advocate of the Tarskian solution is to propose that $(\gamma)$ is not expressible on any level in the Tarskian hierarchy. But, of course, if that's the case, the Tarskian hierarchy hardly reflects the semantics of English. After all, we are able to refer to levels within English. Thus, Tarski's solution is not a solution to the liar as expressed in English. ${ }^{12}$ Further, the Tarskian solution must admit the possibility of referring to, and quantifying into, levels if the theory itself is to be expressible. After all, the solution is committed to the claim that 'Every language level $n$ possesses a particular truth predicate $T_{n}$ '. Thus, either the theory must admit that claims contained within its own theory are not expressible, or that there are versions of the paradox unsolved. As Priest (2006b: p. 263) notes, non-dialetheic solutions "face the dilemma of the choice between inconsistency [admitting true contradictions] and a self-refuting inexpressibility." In comparison, according to Priest, the dialetheic solution to the liar sentences is not incomplete-a unified solution can be provided, by proposing that all of the varieties do indeed entail true contradictions.

\footnotetext{
12 Tarski himself seems to admit this, by accepting both that natural-languages are semantically closed (Tarski 1944: p. 349), and that any attempt to introduce a hierarchy of languages into a given language would be to admit that the language wasn't natural, but artificial (Tarski 1956: p. 267).
} 
Lastly, Priest complains that in addition to restricting the expressiveness of our natural languages, and failing to provide a universal solution to the liar paradoxes, non-dialetheic solutions tend to be ad hoc. That is, they fail to possess any independent motivation for their putative solution. When faced with recalcitrant evidence, we shouldn't favour any fix whatsoever, for "it is not in doubt that we can avoid the paradoxes if we can make any move we like" (Priest 2006b: p. 14). What we are interested in, instead, is a solution which is able to identify a fault in the argument which we have independent reasons to admit. It's clear that the Tarskian solution fails to meet this criterion. The putative solution is simply an attempt to block the contradiction that follows from the liar sentences, and aims to achieve this by supposing that any language must actually contain a hierarchy of languages (and truth predicates), at the cost of deeming many meaningful natural-language claims meaningless. A claim we have no independent motivation for. ${ }^{13}$ According to Priest, the dialetheic solution fails to suffer from this fault. By recognising that we have excellent reasons to accept the semantic closure of natural languages, and the applicability of the T-schema to natural-language sentences within non-opaque settings, we have good reason to lay the blame at the door of classical logic. Particularly, we have good reason to simply admit the truth of the paradoxical conclusion of the liar argument, and thus recognise there are true contradictions. The viability of this solution is only further evidenced by the fact that it (putatively) offers a unified solution to the liar paradoxes.

\subsection{The Russell paradox}

Unlike the liar paradoxes, which are primarily a consequence of fundamental semantic features of natural languages, ${ }^{14}$ the Russell paradox arises as a consequence of the axioms of naïve set theory. Particularly, the Axiom of Comprehension, and generally uncontested logical laws.

According to the original (naïve) account of sets, devised by Cantor and given axiomatic expression by Frege (1950), there are two principles that define the nature of sets: extensionality and comprehension. According to the axiom of extensionality,

(AxEx) $\forall x(x \in y \equiv x \in z) \rightarrow y=z$

two sets are identical if and only if they possess exactly the same members. In other words, the identity of sets is defined exclusively in terms of their membership. The axiom of comprehension, in comparison,

(AxCom) $\exists y \forall x(x \in y \equiv \varphi(x))$ [with only $x$ free in $\varphi$ ]

states that any collection of objects fulfilling a given property constitutes a set.

A lot of hope was placed on these mathematical objects so defined: that they could be used to provide a precise account of infinity, and define other mathematical objects,

\footnotetext{
13 This criterion for a successful solution to the liar paradoxes is not only recognised by dialetheists. Kirkham (1992: p. 281) in his introduction to theories of truth, for example, emphasises the importance of a principled solution, and criticises the Tarskian solution on just this score.

${ }^{14}$ For the reasons given above, versions of the liar can be provided within any semantically closed language - natural or not. However, the dialetheist's arguments, with her appeal to the meaningfulness of certain natural-language sentences and the expressibility of natural-languages generally, are concentrated towards versions of the liar expressed in natural languages.
} 
such as the integers. Unfortunately, the optimism surrounding naïve set theory was very suddenly deflated when Russell (1967) showed that the theory was inconsistent, by sanctioning a set which entailed a contradiction:

(R) $\exists y \forall x(x \in y \equiv x \notin x)$

The set of all sets which are not members of themselves. Using the Russell set (R), only a few steps are required to show that naïve set theory is inconsistent:

\begin{tabular}{ll}
\hline$\exists y \forall x(x \in y \equiv \varphi(x))$ [with only $x$ free in $\varphi]$ & (R1-Axiom of Comprehension) \\
$\exists y \forall x(x \in y \equiv x \notin x)$ & (R2-Substitution of Russell set for $\varphi(x))$ \\
$\exists y(y \in y \equiv y \notin y)$ & (R3-From R2 by Universal Instantiation) \\
$\exists y(y \in y \wedge y \notin y)$ & (R4-From R3 by cases and adjunction) \\
\hline
\end{tabular}

Unlike with versions of the liar, there is almost universal acceptance of a solution to the Russell paradox by philosophers and practicing mathematicians alike. We ought to replace (AxCom) with a more restrictive principle on set construction, such as the Axiom of Specification in Zermelo-Fraenkel set theory with the Axiom of Choice (ZFC):

(AxSpec) $\forall x \exists y \forall z(z \in y \equiv(z \in x \wedge \varphi(z)))$ [with $y$ not free in $\varphi$ ]

Thus, (AxSpec) requires that for any new set $y$ constituted of $z$ f fulfilling condition $\varphi$, the $z$ s must be taken from an already recognised set $x$. (AxSpec) blocks the contradiction flowing from $(\mathrm{R})$ by disallowing the set's formation, as there can be no set of all sets (whether this is the universal set $\mathrm{U}$, or the set of all sets that are not members of themselves).

While recognising that this response from the working mathematician is understandable, Priest (2006b: p. 30) argues that blocking the contradiction in R4 by restricting what was a fundamental principle of naïve set theory is philosophically unacceptable. Rather, we ought to accept the results of our initial postulation, and admit that set theory entails a true contradiction. His reasons for this dialetheic conclusion share similarities to those given for a dialetheic solution to the liar paradoxes, and are fourfold.

Firstly, Priest proposes that (AxCom) is fundamental to the concept of a set; at one stage even suggesting that the axiom partially "analytically characterise [s] the notion of set" (2006b: p. 30). To replace (AxCom) with the more restrictive (AxSpec) is just to admit that not "all conditions do define sets", and that our initial account of what defined this special type of objects we call 'set' isn't even plausible (2006b: p. 31). In other words, by rejecting (AxCom) because of the inconsistency produced by the Russell set, we are not rescuing set theory, but rather getting rid of sets altogether, in favour of some other type of mathematical object.

Secondly, Priest argues that ZFC ultimately relies upon naïve set theory. For to show that an object fulfils the more restrictive (AxSpec), rather than just (AxCom), requires nothing more than to show it appears within the cumulative hierarchy. The cumulative hierarchy of sets is the product of a transfinite recursion which begins with a base case $B$ (whether pure or impure). From the base case, we build the next level in the hierarchy by taking the powerset of $B$, and so on. Thus, letting $\mathrm{V}_{\alpha}$ stand for the 
set in the hierarchy at level $\alpha$ (known as its rank), for any ordinal $\alpha, \mathrm{V}_{\alpha+1}=\wp\left(\mathrm{V}_{\alpha}\right){ }^{15}$ Consequently, in order to be able to express the cumulative hierarchy, and refer to its ranks, we need to presuppose the sense of the ordinals. Yet, as Priest (2006b: p. 31) remarks, the ordinals are defined in set-theoretic terms. Thus, in order to define the ordinals, and thus the cumulative hierarchy, we must already have in mind a notion of set. This obviously cannot be those sets defined within ZFC, as they rely upon the cumulative hierarchy for their own articulation (and the same holds for any other set theory that presupposes the cumulative hierarchy to define sets). Rather, the notion of set presumed must be independent of the cumulative hierarchy, and the most obvious candidate here, according to the Priest (2006b: p. 31), is that of naive set theory. ${ }^{16}$

Thirdly, as with non-dialetheic solutions to the liar paradoxes, restrictions to (AxCom) are criticised for being wholly ad hoc (Priest 2006b: p. 31). The replacement principles were introduced piecemeal simply to avoid the paradoxical results, without independent motivation. In so doing, according to Priest, we run the risk of ignoring the initial motivations for naïve set theory, and accepting another theory which is solely justified by its lack of one particular paradoxical property (whatever the cost). Zermelo, in other words, simply wanted to avoid the paradoxical result so that mathematicians could carry on with their business. ${ }^{17}$

Lastly, Priest (2006b: pp. 32-5) proposes that ZFC does not even allow mathematicians to just get on with their work, for it actually precludes important practice within other areas of mathematics; particularly, category theory. In wishing to speak about mathematical objects in their greatest generality, and perform operations upon these types of objects, category theorists universally quantify over these types. Accordingly, when considering sets, they wish to speak of 'all sets'. Yet, according to the cumulative hierarchy, and thus ZFC, there is no such object as the collection of all sets. After all, there is no universal set. ${ }^{18}$ Thus, rather than allowing the practicing mathematician to get on with their business, ZFC's restricted notion of set blocks our ability to make sense of the category theorist's work in set-theoretic terms.

\subsection{Priest's method of argument}

We've now considered Priest's arguments for why we ought ultimately to accept dialetheism, on the basis of the weaknesses of non-dialetheic solutions to two self-

\footnotetext{
15 Once we reach limit ordinals $\beta$, our definition of sets at a rank changes (for obvious reasons) to: $\mathrm{V}_{\beta}=$ $\bigcup_{\delta<\beta} \mathrm{V}_{\delta}$. However, this detail isn't important for our purposes here.

16 Strictly speaking, all that is required is that the set theory is not well-founded, and naïve set theory is not the only non-well-founded theory available (see, for example, Quine's (1937) NF). However, Priest's presumption here seems to be that naïve set theory should take precedence over other non-well-founded set theories for independent reasons.

17 Priest could have appealed here to Zermelo's (1967: 189) claim that: "Principles must be judged from the point of view of science, and not science from the point of view of principles fixed once and for all." What fundamentally matters is finding axioms that allow mathematicians to continue using set theory, ultimately allowing scientists to rely upon it. Priest's claim is that this is a wholly unprincipled means to justify one's mathematical commitments.

18 Priest was certainly not the first to make this point. It is recognised in Eilenberg and MacLane's (1945: 239 \& 246) original paper, and subsequently by John Bell (1981: 356), who put the point most eloquently.
} 
referential paradoxes: the liar and Russell. The question now is, what conclusions can we draw regarding logical practice from these arguments?

First and foremost, it's clear Priest is presuming that logical theories have a responsibility to provide a solution to certain puzzles, of which the semantic and set-theoretic paradoxes are paradigm examples. Further, that the putative solutions to these puzzles ought to have independent motivation, not simply be an attempt to avoid the unsavoury consequences of the puzzle for the relevant theory. Examples of such a principled and independent motivation would include providing a unified solution to the recognised puzzles, or showing that one's solution respects other important commitments we hold. Inversely, showing that a putative solution to some puzzle fails to provide a unified solution to the recognised puzzle, and does not respect important further commitments, provides evidence that the solution is ad hoc.

We find just such arguments against the principled nature of non-dialetheic solutions to the liar and Russell paradoxes, and for the principled nature of the dialetheic solution within Priest's (2006b) In Contradiction. Non-dialetheic solutions to the liar, for example, fail to respect the meaningfulness of certain natural-language sentences, while the ZFC solution to the Russell paradox neglects the important constitutive principles of sets, and in so doing ensures that we cannot make sense of the practice of mathematicians in certain other areas of mathematics. Dialetheic solutions, in contrast, suffer none of these faults while showing that both of the paradoxes can be solved by the same means - accept their initially paradoxical conclusions and admit that certain contradictions are true.

Consequently, within his arguments, Priest makes at least five methodological assumptions:

1. Solutions to semantic and mathematical puzzles can form part of the explanandum of a logical theory, and thus the relative success of a logical theory at providing a solution can rationally impact logical theory choice.

2. The success at providing such a solution should be judged (at least partially) by the extent to which the solution is independently motivated (and, thus, not ad hoc).

3. Criteria for the independent motivation for a solution include: the solution's ability to solve other puzzles (the solution's unificatory power), and the solution's ability to account for important further commitments.

4. Commitments which would be taken into account when evaluating the viability of a logical theory's solution to the puzzles, and thus the (partial) viability of the logical theory overall, include: the meaningfulness of certain natural-language sentences, the constitutive properties of certain mathematical concepts, and the soundness of certain mathematical results and practices.

5. As a consequence, judgements about the meaningfulness of certain naturallanguage sentences, the constitutive properties of certain mathematical concepts, and the soundness of certain mathematical results and practices, all constitute possible sources of evidence for and against a logical theory.

The important question now is, can Priest's arguments and methodological assumptions be accounted for by the two historically prevalent epistemologies of logic, logical rationalism and semanticism, or do these arguments point in the direction instead of logical abductivism? 


\section{Can rationalism or semanticism explain priest's methodology?}

How good of a fit are logical rationalism or semanticism for Priest's arguments for dialetheism based upon the liar and Russell paradoxes? Let's begin with the argument based upon the liar paradoxes.

While Priest (2006b: p. 17) does propose that the T-schema accurately characterises the truth predicate, this claim is not based on an appeal to definitions or intuitions. Rather, it is a recognition that failures to admit the unrestricted T-schema would unnecessarily restrict the use we put the truth predicate to in natural languages. Further, Priest's claim that it is unreasonable to solve the paradox by denying the semantic closure of natural languages is not based upon any definition of what a natural language is, or an appeal to intuitions. Instead, it's based upon empirical linguistic evidence. It's true that Priest does at times appeal to the meaningfulness of certain natural-language sentences, and that these judgements could easily be construed as intuitions. However, such intuitions are only part of the picture. The appeal to semantic intuitions serve to show that non-dialetheic solutions require us to give up too much which is theoretically important to block the inference to true contradictions. This is no simple appeal to intuitions, or definitions.

In contrast to Priest's argument based upon the liar, the argument based upon the Russell paradox does have more of an air of analyticity about it. Priest (2006b: p. 30) states, for example, that both (AxCom) and (AxExt) "analytically characterise the notion of set." However, when actually arguing against an alternative axiomatic theory of sets, ZFC, Priest does not rely upon simple analytic considerations as one would expect given this admission. Rather, Priest attacks (what he takes to be) naïve set theory's sole competitor in contemporary set theory, demonstrating that current attempts to restrict (AxCom) using the cumulative hierarchy are both unprincipled and, ultimately, presuppose the axiom's use in order to define the cumulative hierarchy. This is a good example of the importance of looking at what practitioners $d o$, rather than simply what they say, when attempting to identify logical practice.

Further, while Priest (2006b: p. 30) suggests as a starting point that our intuitive notion of a set is properly expressed by the combination of (AxCom) and (AxExt), he does not argue that these intuitions directly establish the truth of dialetheism. They merely serve to show that competing set theories must be independently motivated. Far greater emphasis is placed on the claim that even competitors of naïve set theory, which replace (AxCom) with a more restricted principle, end up presupposing the intuitive concept of a set (by using ordinals within the cumulative hierarchy), and thus undermine the goal of these alternative set theories. Thus, at the core of Priest's arguments is the putative inability of non-dialetheic solutions to provide an independently motivated and wholly successful solution to a theoretical puzzle.

Both logical semanticism and rationalism, then, fail to do service to the richness of the arguments found within Priest's case for dialetheism, and a dialetheically suitable logic. There is no simple appeal to definitions or intuitions. Rather, front and centre is placed the importance of logical theories' ability to solve recognised problems, which can take the form of semantic and mathematical paradoxes. As a result, the arguments fit much better the picture of logical epistemology offered up by logicial abductivism. These arguments for dialetheism, and thus a dialetheically suitable logic, are built 
around the question of which logic, treated as a whole, best accommodates certain important data. As we've seen, these relevant data sometimes include conceptual intuitions and the meaning of important mathematical terms, but also puzzles resulting from past logical and mathematical postulations, linguistic judgments, and successes within the mathematical sciences. Further, in accounting for this data, importance is placed upon the theories' abilities to exemplify unificatory power in their solutions to puzzles, and failure to appeal to ad-hoc fixes.

Thus, taken as a piece of logical practice, Priest's arguments for dialetheism based upon the self-referential paradoxes provide some support for logical abductivism. Additionally, the arguments point towards some of the types of data which logics ought to be accommodating if we accept such an abductivism framework for logical epistemology, including: logical paradoxes, linguistic judgments, and successes within the mathematical sciences.

\section{Expanding the search}

Before we conclude, some cautionary words about what we have established, and some objectives for future enquiry, are necessary. So far, all that Priest's arguments for dialetheism tell us is that some form of logical abductivism fits the content of these arguments better than logical rationalism and semanticism. It does not provide us with comprehensive support for logical abductivism as an epistemology of logic. Even if we accept the arguments from Sect. 3 that we ought to allow the practice of experts within their field to direct our theory of epistemology for that field (and we think we ought to accept them), Priest's arguments are only a small sample, and many more cases need to be considered before we can take logical practice to provide significant evidence for any particular epistemology of logic. After all, we may find that Priest's arguments are wholly unrepresentative of those normally found within logical practice. However, by the same token, it is important not to dismiss lightly arguments found within the literature. Generally, for arguments within the literature to be disregarded, and not taken to constitute evidence for a theory of logical epistemology, it is not sufficient that they are found to be inadequate as arguments for their proposed position. We do not require our practitioners to be infallible. Instead, to deem an argument irrelevant to a theory of logical epistemology, it would have to be shown that the types of evidence proposed in the argument were wholly unsuitable, and this can only be done by considering the attitudes of others within the community to it. A flawed or inadequate argument is not the same as a wholly unsuitable one. Only when an argument is shown to be of the latter type should we disregard it as a piece of data to be accommodated by a theory of logical epistemology.

One may be concerned that in looking to practice, and finding practice that motivated logical abductivism, we used as a case study the arguments of an avowed logical abductivist (Priest). That, in some sense, this choice of case study unjustifiably prejudiced logical abductivism from the start. ${ }^{19}$ While it is only natural that one should be wary of appealing to the practice of a logician to justify a particular theory of logical

$\overline{19}$ This concern was raised by an anonymous referee. 
methodology if that same logician has admitted endorsing the theory, any concern that this choice unjustifiably favoured logical abductivism is ultimately misplaced. The only reason that a choice of case study could unjustifiably prejudice a particular theory of logical methodology over others is if the practice was in some sense inappropriate, or unrepresentative of logical practice as a whole (in this case, due to the practitioner's philosophical tastes). In other words, that the philosophical views of the logician have distorted proper logical practice. After all, the philosophical views of the logician will not matter unless it unduly affects their practice, which subsequently affects the results of the practice-based approach. Yet, the practice-based approach has safeguards to ensure that the case studies it considers do not deliver unjustifiably prejudiced results.

Firstly, as practice is peer evaluated, if the logician's arguments are inappropriate in any sense (note, not just bad, but inappropriate), then this will come out either through initial peer review or subsequent discussion of the faulty methodology. Thus, any possible prejudice towards a particular methodology of logic, whatever the cause, is safeguarded against on the whole by the community. Of course, it may be that Priest's arguments are deemed to be inappropriate by peers, but this will only be shown by first considering Priest's practice as a case study and then detailing instances where such practice has been considered to be deviant by the community. Until we have any such reasons to believe this practice is deviant, given that the relevant arguments passed through peer review, there must be the assumption that they are examples of good practice, and thus that they shouldn't be discounted as suitable data to be studied by the practice-based approach, regardless of the logician's philosophical views.

Secondly, the practice-based methodology is not only protected against the potential deviance of a logician's practice by the reactions of their peers, but through the consideration of further case studies. As stated above, no one case study will provide significant evidence for a particular theory of logical methodology. The case for a theory must be built up over time using multiple case studies. Further, by using numerous case studies, outliers and unusual practice can then be appropriately identified. However, these case studies should include the most significant theories/arguments within the literature (see Burian 2001), and Priest's arguments for dialetheism definitely fit that bill. Thus, not only should we have no fears in using Priest's practice in order to evaluate the available theories of logical methodology, but we have excellent reason to consider Priest's arguments as a case study, as part of the overall picture.

So much for the cautionary words. Where can we look to next for further evidence, and to put the suitability of Priest's arguments to the test? Well, firstly, we should look to the motivating arguments of those other great non-classical research programmes: intuitionistic, relevant, substructural, and paracomplete logics. Secondly, however, we must look at the responses to these arguments. These replies are often revealing, both in the potential validation of good practice they provide the initial arguments (thereby answering the concerns voiced above), and in their expression of the complex dynamics between logical positions.

Take, for example, the wide range of responses to dialetheism found within the literature. As the disagreement between dialetheists and non-dialetheists isn't a simple choice between two logics, there are three broad types of responses to dialetheism available for the non-dialetheist. Those that aim to: 
Attack the Hardcore. Replies which argue that dialetheism is fundamentally incoherent, or that no dialetheically suitable logic possesses a property that any viable logic must possess. If successful, such arguments would show that dialetheic interpretations of these logics are precluded, given that dialetheism is incoherent, or that dialetheically suitable logics as a whole are unviable. Examples include:

- Dialetheism contravenes the definition of 'contradiction'. (Slater 1995)

- Admitting a contradiction ensures that all science would collapse. (Popper 1940: p. 410)

Undermine the Hardcore. Replies which propose that none of the dialetheic arguments are well-founded. If successful, such arguments would only show that dialetheic interpretations of dialetheically suitable logics are unsubstantiated, and not unviable. Examples include:

- Dialetheically suitable logics suffer just as much from revenge paradoxes as nondialetheic logics. (Littmann and Simmons 2004; Scharp 2013: Ch. 4)

- Dialetheic arguments appeal to inappropriate vernacular data. (Tkaczyk 2016)

Attack the Softcore. Replies proposing that some present dialetheically suitable logics fail to have a certain property which any adequate logic should possess. The impact of such arguments, if successful, is less certain. Such impact, in part, would depend upon the strength of the motivations for dialetheism, the importance of the property which the logics fail to possess, and the prevalence of the problem among logics (whether dialetheically suitable or not). Examples include:

- Dialetheically suitable logics cannot accommodate important mathematical theories. (Tennant 2004)

- One cannot express disagreement with dialetheic semantics. (Parsons 1990)

- Dialetheically suitable logics (in conjunction with dialetheism) entail that the actual world is impossible. (Martin 2015)

- Dialetheic semantics cannot express meaningful natural-language sentences. (Shapiro 2004)

These replies to dialetheism deserve their own treatment, and in so doing we should get a better sense of how widely shared the criteria for logical evidence found within Priest's arguments are. The prospects of finding such shared methodological principles are certainly promising. For example, there is little to no call within the literature to disregard troublesome natural-language cases, such as the liar, as irrelevant to the business of logic (Eklund 2002). Further, it's recognized elsewhere by those opposed to a dialetheic solution that one of the goals of a logical theory is to provide a unified and non-ad hoc solution to logico-semantic puzzles (Littmann and Simmons 2004), and that logics should not deem meaningless what are meaningful natural-language sentences (Shapiro 2004). A proper, and thorough, investigation of these responses should bring out the nuanced points of methodological (dis)agreement between those involved in the debate. 


\section{Conclusion}

This paper tasked itself with two primary goals. Firstly, to demonstrate the fruitfulness of using logical practice to inform a theory of logical epistemology. This goal we take the paper to have met without question. Not only are there good independent reasons to believe that our theories of logical epistemology should consider logical practice, based upon precedent from the natural sciences and mathematics, but even a brief consideration of two prevalent arguments within logic show that we can discover much about the methodological assumptions of logicians from their arguments.

Secondly, the paper aimed to show how certain prevalent arguments within the logical literature lend support to logical abductivism over competing theories of logical epistemology, while also providing details on the types of data logics are concerned with accommodating. This goal has also been met. We have seen how the dialetheist's arguments based upon the self-referential paradoxes fail to concern themselves with definitions or intuitions wholly. Rather, a more diverse set of reasons are given for endorsing one theory of validity over another, including their ability to solve logicosemantic puzzles, accommodate the meaningfulness of natural-language sentences, and respect practice within the mathematical sciences. To confirm the results from this case study, and show that logical abductivism is indeed the epistemology of logic presupposed by logicians within their practice, further prevalent cases of logical debate must be analysed, with particular attention paid to replies to dialetheism. That is a task for future research.

Acknowledgements Open Access funding provided by University of Bergen. I am grateful to colleagues at the University of Bergen for their comments on a draft of this paper, particularly Pål Antonsen, Sorin Bangu, Ole Hjortland, Tore Øgaard, and Sindre Søderstrøm. I would also like to thank Graham Priest and two anonymous referees for their detailed comments on a previous version of this paper.

Funding Research for this paper was supported by a European Research Council (ERC) Grant (No: 797507), under the European Union's Horizon 2020 research and innovation programme.

Open Access This article is licensed under a Creative Commons Attribution 4.0 International License, which permits use, sharing, adaptation, distribution and reproduction in any medium or format, as long as you give appropriate credit to the original author(s) and the source, provide a link to the Creative Commons licence, and indicate if changes were made. The images or other third party material in this article are included in the article's Creative Commons licence, unless indicated otherwise in a credit line to the material. If material is not included in the article's Creative Commons licence and your intended use is not permitted by statutory regulation or exceeds the permitted use, you will need to obtain permission directly from the copyright holder. To view a copy of this licence, visit http://creativecommons.org/licenses/by/4.0/.

\section{References}

Ayer, A. J. (1936). Language, truth and logic. New York, NY: Dover.

Batens, D., \& De Clercq, K. (2004). A rich paraconsistent extension of full positive logic. Logique et Analyse, 47(185/188), 227-257.

Bealer, G. (1998). Intuition and the autonomy of philosophy. In M. DePaul \& W. Ramsey (Eds.), Rethinking intuition: The psychology of intuition and its role in philosophical inquiry (pp. 201-240). Lanham: Rowman \& Littlefield.

Beall, Jc (2009). Spandrels of truth. Oxford: Clarendon Press. 
Bell, J. (1981). Category theory and the foundations of mathematics. British Journal for the Philosophy of Science, 32(4), 349-358.

Boghossian, P. A. (1996). Analyticity reconsidered. Nô̂s, 30(3), 360-391.

Boghossian, P. A. (2000). Knowledge of logic. In P. A. Boghossian \& C. Peacocke (Eds.), New essays on the a priori (pp. 229-254). Oxford: Clarendon Press.

Boghossian, P. A. (2001). Inference and insight. Philosophy and Phenomenological Research, 63(3), 633-640.

BonJour, L. (1998). In defense of pure reason. Cambridge: Cambridge University Press.

Brown, B. (1999). Yes, virginia, there really are paraconsistent logics. Journal of Philosophical Logic, 28(5), 489-500.

Burian, R. M. (2001). The dilemma of case studies resolved: The virtues of using case studies in the history and philosophy of science. Perspectives on Science, 9(4), 383-404.

Carnap, R. (1937). The logical syntax of language. New York, NY: Routledge \& Kegan Paul Ltd.

Carnap, R. (1963). The philosophy of Rudolf Carnap. Cambridge: Cambridge University Press.

Carroll, L. (1895). What the tortoise said to achilles. Mind, 4(14), 278-280.

Chudnoff, E. (2011). What intuitions are like. Philosophy and Phenomenological Research, 82(3), 625-654.

Curry, H. B. (1942). The inconsistency of certain formal logics. Journal of Symbolic Logic, 7(3), 115-117.

da Costa, N. C. A. (1974). On the theory of inconsistent formal systems. Notre Dame Journal of Formal Logic, 15(4), 497-510.

da Costa, N. C. A. (1982). The philosophical import of paraconsistent logic. Journal of Non-classical Logic, 1(1), 1-19.

Dunn, J. M. (1976). Intuitive semantics for first-degree entailments and 'coupled trees'. Philosophical Studies, 29(3), 149-168.

Eilenberg, S., \& MacLane, S. (1945). General theory of natural equivalences. Transactions of the American Mathematical Society, 58(2), 231-294.

Eklund, M. (2002). Inconsistent languages. Philosophy and Phenomenological Research, 64(2), 251-275.

Field, H. H. (2008). Saving truth from paradox. Oxford: Oxford University Press.

Frege, G. (1950). The foundations of arithmetic, J. L. Austin (trans). Oxford: Blackwell.

Frege, G. (1952). In P. Geach \& M. Black (Eds.), Translations from the philosophical writings of Gottlob Frege. Oxford: Basil Blackwell.

Frege, G. (1965). The basic laws of arithmetic, M. Furth (trans). Berkeley, CA: University of California Press.

Giaquinto, M. (2007). Visual thinking in mathematics. Oxford: Oxford University Press.

Giaquinto, M. (2008). The linguistic view of a priori knowledge. Philosophy, 83(1), 89-111.

Hacking, I. (1983). Representing and intervening. Cambridge: Cambridge University Press.

Hjortland, O. T. (2017). Anti-exceptionalism about logic. Philosophical Studies, 174(3), 631-658.

Jaśkowski, S. (1969). Propositional calculus for contradictory deductive systems. Studia Logica, 24(1), $143-157$.

Kabay, P. D. (2008). A defense of trivialism (PhD thesis). University of Melbourne, Australia.

Kirkham, R. L. (1992). Theories of truth. Cambridge, MA: MIT Press.

Kripke, S. (1975). Outline of a theory of truth. Journal of Philosophy, 72(19), 690-716.

Littmann, G., \& Simmons, K. (2004). A critique of dialetheism. In G. Priest, J. Beall, \& B. Armour-Garb (Eds.), The law of non-contradiction: New philosophical essays (pp. 314-335). Oxford: Clarendon Press.

Martin, B. (2015). Dialetheism and the impossibility of the world. Australasian Journal of Philosophy, 93(1), 61-75.

Martin, B. (2018). In defence of dialetheism: A reply to Beziau and Tkaczyk. Logic and Logical Philosophy, 27(2), 205-233.

Martin, B., \& Hjortland, O. T. (2020). Evidence in logic. In M. Lasonen-Aarnio \& C. Littlejohn (Eds.), Routledge handbook for the philosophy of evidence. London: Routledge.

Mayo, D. G. (1991). Novel evidence and severe tests. Philosophy of Science, 58(4), 523-552.

McGee, V. (1991). Truth, vagueness, and paradox. Indianapolis, IN: Hackett.

Parsons, T. (1990). True contradictions. Canadian Journal of Philosophy, 20(3), 335-354.

Peregrin, J., \& Svoboda, V. (2017). Reflective equilibrium and the principles of logical analysis: Understanding the laws of logic. London: Routledge.

Popper, K. R. (1940). What is dialectic? Mind, 49(196), 403-426.

Priest, G. (1979). Logic of paradox. Journal of Philosophical Logic, 8(1), 219-241. 
Priest, G. (2006a). Doubt truth to be a liar. Oxford: Clarendon Press.

Priest, G. (2006b). In contradiction: A study of the transconsistent (2nd ed.). Oxford: Clarendon Press.

Priest, G. (2014). Revising logic. In P. Rush (Ed.), The metaphysics of logic (pp. 211-223). Cambridge: Cambridge University Press.

Priest, G., \& Routley, R. (1989). Systems of paraconsistent logic. In G. Priest, R. Routley, \& J. Norman (Eds.), Paraconsistent logic: Essays on the inconsistent (pp. 151-186). München: Philosophia Verlag.

Quine, W. V. (1937). New foundations for mathematical logic. American Mathematical Monthly, 44(2), $70-80$.

Quine, W. V. (1951). Two dogmas of empiricism. Philosophical Review, 60(1), 20-43.

Quine, W. V. (1986). Philosophy of logic (2nd ed.). Cambridge, MA: Harvard University Press.

Russell, B. (1920). Introduction to mathematical philosophy (2nd ed.). London: George Allen and Unwin.

Russell, B. (1957). On scientific method in philosophy. Mysticism and logic (pp. 93-119). New York, NY: Doubleday.

Russell, B. (1967) [1902]. Letter to Frege. In J. Van Heijenoort (Ed.), From Frege to Gödel: A source book in mathematical logic, 1879-1931 (pp. 124-125). Cambridge, MA: Harvard University Press.

Russell, G. (2014). Metaphysical analyticity and the epistemology of logic. Philosophical Studies, 171(1), $161-175$.

Russell, G. (2015). The justification of the basic laws of logic. Journal of Philosophical Logic, 44(6), 793-803.

Scharp, K. (2013). Replacing truth. Oxford: Oxford University Press.

Shapiro, S. (2000). The status of logic. In P. A. Boghossian \& C. Peacocke (Eds.), New essays on the a priori (pp. 333-366). Oxford: Clarendon Press.

Shapiro, S. (2004). Simple truth, contradiction, and consistency. In G. Priest, J. Beall, \& B. Armour-Garb (Eds.), The law of non-contradiction: New philosophical essays (pp. 336-354). Oxford: Clarendon Press.

Slater, H. (1995). Paraconsistent logics? Journal of Philosophical Logic, 24(4), 451-454.

Tarski, A. (1944). The semantic conception of truth and the foundations of semantics. Philosophy and Phenomenological Research, 4(3), 341-375.

Tarski, A. (1956). The concept of truth in formalized languages. In J. Corcoran (Ed.), Logic, semantics and metamathematics: Papers from 1923 to 1938 (trans: Woodger JH (pp. 152-278). Oxford: Oxford University Press.

Tennant, N. (2004). An anti-realist critique of dialetheism. In G. Priest, J. Beall, \& B. Armour-Garb (Eds.), The law of non-contradiction: New philosophical essays (pp. 355-384). Oxford: Clarendon Press.

Tkaczyk, M. (2016). The case of dialetheism. Logic and Logical Philosophy, 25(2), 203-224.

Van Bendegem, J. P. (2003). Thought experiments in mathematics: Anything but proof. Philosophica, 72(2), 9-33.

Van Kerkhove, B., \& Van Bendegem, J. P. (2008). Pi on earth, or mathematics in the real world. Erkenntnis, $68(3), 421-435$.

Williamson, T. (2007). The philosophy of philosophy. Oxford: Blackwell.

Williamson, T. (2017). Semantic paradoxes and abductive methodology. In B. Armour-Garb (Ed.), The relevance of the liar (pp. 325-346). Oxford: Oxford University Press.

Wright, C. (1986). Inventing logical necessity. In J. Butterfield (Ed.), Language, mind, and logic (pp. 187-209). Cambridge: Cambridge University Press.

Zermelo, E. (1967) [1908]. A new proof of the possibility of a well-ordering. In J. Van Heijenoort (Ed), From Frege to Gödel: A source book in mathematical logic, 1879-1931 (pp. 183-198). Cambridge, MA: Harvard University Press.

Publisher's Note Springer Nature remains neutral with regard to jurisdictional claims in published maps and institutional affiliations. 